\title{
Die Regionalpolitik des Bundes
}

\section{Ausgangslage}

Die schweizerische Regionalpolitik steht in engem $\mathrm{Zu}$ sammenhang mit dem föderativen Staatsaufbau und mit der soziokulturellen Vielfalt der Schweiz. Sowohl der Bund als auch Kantone und Gemeinden können regionalpolitische Instrumente einsetzen. Ein Rückblick auf die letzten 30 Jahre zeigt, daß hier - wie in andern Bereichen der schweizerischen Wirtschaftspolitik - zurückhaltend und pragmatisch vorgegangen wird.

Allerdings ist in den letzten Jahren unter dem Eindruck der Globalisierung der Märkte und, gestoßen durch eine animierte Föderalismusdiskussion, die schweizerische Regionalpolitik vermehrt grundsätzlich hinterfragt worden.' Hinzu kommt die Integrationsthematik, welche die schweizerische Politik mit jener der EU in Beziehung setzt.

\section{Darstellung 1}

\section{Regionalpolitische Problemstellungen}

- Interregionale Entwicklungsund Wohlstandsdisparitäten.

- Entleerung und ökologische Bedrohung des Berggebietes sowie des ländlichen Raumes

- Industrielle Problemregionen

- «Regionale Isolation» als Folge der Integrationspolitik

- Entmischung und Ballungskosten

Darstellung $I$ gibt einen groben Überblick über jene Probleme, die für die Regionalpolitik des Bundes im Vordergrund stehen. Trotz Bemühungen, eine schweizerische Raumordnungspolitik zu formulieren, stehen instrumentell das Berggebiet und industrielle Problemregionen nach wie vor im Zentrum. Erste Schritte in Richtung ländlichen Raum werden getan. Praktisch keine unmittelbaren Maßnahmen unternimmt der Bund im Bereich der Stadtpolitik. ${ }^{2}$

Seit vielen Jahren existiert in der Schweiz eine Diskussion darüber, ob die interregionalen Disparitäten zuoder abgenommen haben. Je nach Ansatz und Meßkonzept ändern sich die Antworten, aber mit Bestimmtheit kann festgehalten werden, da $\beta$ angesichts der Kleinheit des Landes, der hohen außenwirtschaftlichen Abhängig- keit und der feinmaschigen Organisation kantonale Volkseinkommenszahlen problematische Indikatoren sind. Die Veränderung der Einkommensdisparitäten (wie übrigens auch jene der Beschäftigung) ist weitgehend zyklisch, konjunkturell beeinflußt. So kann man allein feststellen, daß die Position der Kantone im Laufe der Zeit praktisch gleichgeblieben ist; selbst der Prozeß der Deindustrialisierung vermochte die Rangliste nicht wesentlich zu verändern. Die Auswirkungen des Strukturwandels auf die einzelnen Regionen der Schweiz untersucht neuerdings A. ROSSI. ${ }^{3}$

\section{Ziele und Strategien}

Die Regionalpolitik des Bundes kann - etwas überspitzt formuliert - als ein typisches Kind des Kompromisses umschrieben werden. In einem kürzlich vom Bundesamt für Industrie, Gewerbe und Arbeit veröffentlichten Artikel wurde dies so ausgedrückt:

«Regionalpolitik hat nicht nur einen wesentlich wirtschaftspolitischen, sondern einen ebenso hohen staatsund kohäsionspolitischen Stellenwert» (DIE VOLKSWIRTSCHAFT 1/1995).

Die Regionalpolitik ist zwar Teil der allgemeinen Wirtschaftspolitik des Bundes, soll aber gleichzeitig einen gewissen Ausgleich aus Rücksicht auf den Föderalismus ermöglichen. Impliziert wird also, daß der Wettbewerbsgedanke des föderativen Systems und des Marktes bundesstaatliche Interventionen zur Harmonisierung der «Startchancen» bedingten. Der damit zutage tretende Zielkonflikt zwischen gesamtwirtschaftlicher Effizienz und regionaler Redistribution entlädt sich auch in der Schweiz regelmäßig in harten Grundsatzdiskussionen. Geprägt vom Geist der dreißiger Jahre, spricht Art. $31^{\text {bis }}$ der Bundesverfassung sogar von Maßnahmen «zum Schutz bedrohter Landesteile», und Art. $31^{\text {quinquies }}$ der Bundesverfassung, der sogenannte Konjunkturartikel, postuliert, daß bei der Stabilitätspolitik auf die regionalen Auswirkungen Rücksicht genommen werden müsse. Der Einbezug zusätzlicher Ziele, insbesondere die sparsame Nutzung natürlicher Ressourcen, der ökologische Aspekt also, hat die Aufgabe methodisch sicher nicht erleichtert. Die Auseinandersetzungen um die Alpen-

Stephan Bieri, Dr., Delegierter und Vizepräsident des ETHRats, ETH Zentrum, 8092 Zürich 
konvention zeigen, wie sich ein theoretisches Dilemma unversehen zu einem politischen Zündstoff auswachsen kann. Die Regionalpolitik des Bundes steht im Spannungsfeld vielfältiger, komplexer Anliegen. Um mit dieser Situation einigermaßen fertig zu werden, bedarf es zumindest griffiger Strategien.

\section{Darstellung 2}

\section{Strategien}

- Passive Sanierung

$$
\begin{aligned}
& \text { - Verbesserung der Standortfaktoren } \\
& \text { - Direkte Beeinflussung der Kosten } \\
& \text { - Förderung von Innovation } \\
& \text { und Diversifikation }
\end{aligned}
$$

- Internalisierung der Ballungskosten

Hier äußert sich nun die vom Bund geübte Zurückhaltung: Im Vordergrund stehen Maßnahmen zur Verbesserung der Standortfaktoren (z.B. Infrastrukturpolitik) oder, für Schlüsselbereiche, eine gezielte Beeinflussung der Kosten von Unternehmungen (z. B. Subventionierung von Finanzierungsmaßnahmen). Mittelbar oder unmittelbar einbezogen wird zunehmend auch die Technologiepolitik, die ihrerseits verschiedene Schnittstellen $\mathrm{zu}$ andern Interventionen besitzt. Verschiedene praktische Maßnahmen des Bundes und der Kantone, so etwa die Schaffung von CIM-Zentren und die engere Zusammenarbeit zwischen Universitäten und andern tertiären Bildungsinstitutionen, verschaffen der technologieorientierten Regionalpolitik mehr Druck. Hinzu kommen die Bestrebungen einzelner Kantone (z. B. Neuenburg), weitergehende regionale Vernetzungen - insbesondere industrielle Clusters - zu fördern. ${ }^{4}$

Entsprechend dem früher gemachten Hinweis sind keine expliziten stadtpolitischen Strategieelemente erkennbar; Aspekte der Internalisierung, zum Beispiel im Zusammenhang mit der Mobilität, bleiben der Verkehrspolitik und der kantonalen Initiative überlassen.

\section{Instrumente (Auslegeordnung)}

Bekanntlich besteht ein Unterschied darin, was der Gesetzgeber bezweckt und welches die tatsächlichen Wirkungen der von ihm eingeleiteten Maßnahmen sind. In der Regionalpolitik existieren besonders delikate, fein verästelte Wirkungszusammenhänge; die Inzidenz der Regionalpolitik des Bundes stellt denn auch eine besondere Herausforderung an die empirische Forschung.

In der Folge wird jedoch ein anspruchsloserer, positivistischer Ansatz gewählt. Darstellung 3 gibt eine Übersicht über die wichtigsten regionalpolitischen Instrumente des Bundes so, wie sie in der Praxis eingesetzt werden. Im Vordergrund stehen dabei das Investitionshilfe-

\section{Darstellung 3}

Übersicht über die wichtigsten Instrumente des Bundes

- Bundesgesetz über Investitionshilfe für Berggebiete (IHG), Rev. 1984

- Bundesbeschluß über Finanzierungsbeihilfen zugunsten wirtschaftlich bedrohter Regionen, Rev. 1984, Verl. 1994 / zugunsten wirtschaftlicher Erneuerungsgebiete 1995

- Bundesgesetz über die Förderung des Hotel- und Kurortkredites

- Bundesgesetz über die Bürgschaftsgewährung in Berggebieten

- Raumplanungsgesetz

- Finanzausgleichsgesetz

gesetz (IHG), welches auf das Berggebiet ausgerichtet ist, und der Bundesbeschluß über Finanzierungsbeihilfen zugunsten wirtschaftlicher Erneuerungsgebiete, mit welchem auf Unternehmungsebene klassische Wirtschaftsförderung betrieben werden kann. Strategisch dominieren damit die Ansätze «Verbesserung von Standortfaktoren» und "Beeinflussung von Kosten». Beide Instrumente engagieren direkt die interessierten Kantone - gehen also in Richtung Vollzugsföderalismus. Der hohe Stellenwert des Berggebietes für die Regionalpolitik des Bundes führte schon früh zur Einbindung der Tourismuspolitik und bietet heute verschiedenartige Möglichkeiten der Überlagerung und Ergänzung von Maßnahmen.

Die Gesetzgebung auf dem Gebiet der Raumplanung und des Finanzausgleichs fand in der Vergangenheit immer wieder regionalpolitische Beachtung, namentlich auch im Zusammenhang mit der verwaltungsinternen Koordination. Die besondere systematische Bedeutung dieser zwei Instrumente wird im Zusammenhang mit der Neuorientierung der Regionalpolitik entsprechend gewichtet.

Bundesrat und Parlament haben sich in den letzten Jahren vor dem Hintergrund des strukturellen Wandels der schweizerischen Wirtschaft über neue regionalpolitische Instrumente unterhalten. Aus der recht schwerblütig geführten Diskussion sind schließlich drei Instrumente hervorgegangen, welche den verantwortlichen Behörden von Bund und Kantonen wenigstens eine breitere Mitwirkung auf dem internationalen Parkett ermöglichen (Darstellung 4).

Die Anhänge I und 2 illustrieren die Wirkungsweise und die finanzielle Bedeutung der beiden Hauptinstrumente der Regionalpolitik des Bundes. IHG und Finanzierungsbeihilfen bilden letztlich auch organisatorisch das Rückgrat der Regionalpolitik des Bundes. So hat die Berggebietsförderung für den gesamten gesetzlichen Perimeter umfassende Entwicklungskonzepte hervorgebracht, die nicht nur methodisch, sondern auch für die 


\section{Darstellung 4}

\section{Neue regionalpolitische Instrumente des Bundes}

- Bundesbeschluß über die Förderung der grenzüberschreitenden Zusammenarbeit von Kantonen und Regionen im Rahmen der Gemeinschaftsinitiative Interreg II in den Jahren 1995-1999 (8.3.1995)

- Bundesbeschluß über die Teilnahme an internationalen Informations-, Vermittlungs- und Beratungsprogrammen zugunsten kleiner und mittlerer Unternehmen (6.10.1995)

- Bundesbeschluß zur Förderung der Information über den Unternehmensstandort Schweiz (6.10.1995)

regionale Zusammenarbeit bedeutsam sind. Im Berggebiet und in den industriellen Problemregionen haben die Regionalsekretariate neben den vollziehenden immer mehr gestaltende Aufgaben übernommen. Im internationalen Vergleich ist dies ein sehr wertvolles Erfahrungskapital, dem Sorge getragen werden soll. Allerdings muß in Zukunft ein Weg zur Überwindung der Kleinräumigkeit und zur weitern Unterstützung flexibler Vernetzungen gefunden werden.

\section{Wo stehen wir?}

Die Regionalpolitik des Bundes steht - wie andere Bereiche der Wirtschaftspolitik - unter dem Eindruck einer nur schleppenden konjunkturellen Erholung und der immer tiefere Spuren hinterlassenden Globalisierung. Die Wechselkurssituation trifft sowohl Tourismus als auch Industrieregionen hart. Der Ruf nach "Standortsicherung» und entsprechender regionalpolitischer Flankierung wird laut. Zusätzlicher, kurzfristiger Handlungsbedarf erscheint notwendig wegen haushaltpolitischer Engpässe, infolge bundesweiter Reorganisationen (z. B. Abbau von Arbeitsplätzen in Rüstungsbetrieben), aber auch im Hinblick auf die Auswirkungen der WTO (z. B. Aufhebung regionaler Schutzmechanismen bei öffentlichen Vergaben).

Das europapolitische Abseitsstehen der Schweiz besitzt verschiedene regionalpolitische Dimensionen. Wie immer man die Qualität der Brüsseler Regionalpolitik beurteilt: der Schweiz fehlt für ihre Politik ein größerer Konsolidierungsrahmen. So heißt das Motto zurzeit "to do the best of it», und verschiedene Kantone versuchen mindestens, in ihren Grenzräumen die notwendigen internationalen Kontakte zu intensivieren. Hierbei kommen ihnen die mit dem Bundesbeschluß «Interreg II» gesprochenen Mittel zweifellos gelegen.
Aber es darf nicht übersehen werden, daß sich der schweizerischen Wirtschaftspolitik prinzipielle, auch das Verfassungsverständnis betreffende Fragen stellen. Vor dem Hintergrund der in integrationspolitischen $\mathrm{Ab}$ stimmungen wesentlichen Kräfteverhältnisse (Ständemehr) könnte sogar die Frage aufkommen, ob die bisherigen Geberregionen, die sich in der Regel proeuropäisch gaben, die in der Regionalpolitik geübte Solidarität aufgeben. Bei einer mehr wirkungsorientierten, strukturpolitischen Sichtweise mag man sich auch die Frage stellen, wo die beschränkten Ressourcen in Zukunft schwergewichtig einzusetzen seien. Soll bei einer gesamtwirtschaftlichen Stagnation an einem Ausgleich der Schwachen festgehalten oder, auch aus regionalpolitischem Interesse, auf eine Förderung der Starken eingeschwenkt werden?

Die Diskussion um die Revitalisierung der schweizerischen Volkswirtschaft hat dieses Thema aufgenommen, ohne daß allerdings tiefere empirische Untersuchungen über die maßgeblichen Wirkungszusammenhänge vorlägen. Vor rund drei Jahren hat das Eidgenössische Volkswirtschaftsdepartement ein Projekt «Neuorientierung der Regionalpolitik» lanciert, welches sich auch mit diesem Trade-off auseinandersetzte. Darstellung 5 zeigt stark vereinfacht - die wesentlichen Problemfelder und Schnittstellen.

Durstellung. 5
Neue Akzente

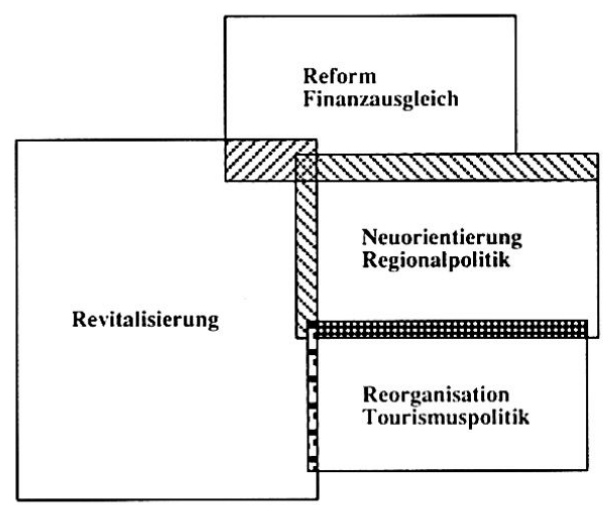

\section{Erneuerungstendenzen}

Die Regionalpolitik des Bundes ist in jüngerer Zeit durch das Nationale Forschungsprogramm 25 und die Aufgabenneuverteilung zwischen Bund und Kantonen positiv beeinflußt worden. Zudem beschäftigen sich verschiedene Organisationen einläßlich mit der Gestaltung der Regionalpolitik. Neben den klassischen Stimmen aus dem Bereich der kantonalen Volkswirtschaftsdirektoren, des 
Tourismus oder der Berggebietsförderung im engen Sinn (z. B. SAB) ist auf ein Manifest der schweizerischen Studiengesellschaft für Raumordnung und Regionalpolitik (ROREP) hinzuweisen. ${ }^{5}$ Als wesentlichste Forderung ist die Schaffung variabler Bezugsgebiete - das heißt eine "géométrie variable» - zu erwähnen.

Die bundesrätliche Beratende Kommission für regionale Wirtschaftsförderung ergriff bereits Ende der achtziger Jahre eine Initiative, die Regionalpolitik des Bundes selektiver und flexibler zu gestalten. Gemeinsam mit dem Bundesamt für Industrie, Gewerbe und Arbeit hat sie das Projekt «Neuorientierung der Regionalpolitik» getragen. Im Mai 1994 verabschiedete sie einen Grundsatzbericht, der vier Reformtendenzen betonte. Erstens soll die Regionalpolitik bewußt gesamtwirtschaftlich eingebettet werden. Zweitens schlägt die Kommission insofern eine Kurskorrektur vor, als in Zukunft das Gewicht mehr auf Maßnahmen der Allokationseffizienz denn der Umverteilung zu legen sei. Drittens befürwortet sie - übrigens in Übereinstimmung mit dem Bundesamt für Konjunkturfragen - eine konsequente Einbindung von Forschung, Technologie und Bildung in die Regionalpolitik. Und viertens unterstützt sie alle Bestrebungen, die ein stärkeres Engagement der Kantone zur Folge haben. Eine wichtige Voraussetzung für eine in diesem Sinn schlankere Regionalpolitik stellt, wie bereits angedeutet, die vorgesehene Revision des bundesstaatlichen Finanzausgleichs dar. Die Förderung des nicht zweckgeb ndenen, freien Finanzausgleichs als Absicherungsmittel in einem föderativen System ist allerdings nicht nur für die Regionalpolitik, sondern ebenso für eine Entflechtung der Zuständigkeiten und die Haushaltssanierung bedeutsam. ${ }^{6}$ Dieser Zusammenhang wiederum erlaubt es auf die Dauer nicht, die Neuorientierung der Regionalpolitik unabhängig von andern Reformen durchzuspielen.

Bundesrat und Parlament sind auf diese Vorschläge teilweise eingegangen. In der Auseinandersetzung zwischen ordnungspolitischer Grundsatzkritik (insbesondere seitens der Spitzenverbände der Wirtschaft), Besitzstandsdenken und wissenschaftlicher Herausforderung ist der schließlich getroffene Kompromiß sicher akzeptabel. Indessen ist anzunehmen, daß längerfristig der strukturpolitische Druck nicht abnimmt, was allenfalls zu neuen Interventionswünschen und Belastungen führen kann.

Darstellung 6 zeigt jene regionalpolitischen Schritte, die als Folge der Neuorientierung zu erwarten sind.

Die vom Bundesrat am 28. Februar 1996 verabschiedete Botschaft über die Neuorientierung der Regionalpolitik soll «die Wettbewerbsfähigkeit und eine nachhaltige Entwicklung in den Teilgebieten unseres Landes fördern und zur Erhaltung der dezentralen Wohnortsqualität beitragen».' Die Hauptstoßrichtung der Revision des IHG ist eine sukzessive Abkehr von der "Verteilungsorientierung», wobei gleichzeitig eine vermehrte Förderung der regionalen Entwicklungsvoraussetzungen angestrebt wird. Mit dem Entwurf des Bundesbeschlusses «Regio plus» soll der sich im ländlichen Raum vollziehende Strukturwandel begleitet und die Entwicklung der vor-
Darstellung 6

\section{Nächste Schritte}

- Auswertung des Vernehmlassungsverfahrens «Neuorientierung Regionalpolitik»

- Vorlagen an die eidgenössischen Räte - Revision IHG

- Bundesbeschluß über Maßnahmen zur Flankierung des Strukturwandels im ländlichen Raum («Regio plus»)

- Organisatorische Straffungen und Maßnahmen im Zusammenhang mit der parlamentarischen Verwaltungskontrolle (Thema «Koordination»)

handenen Potentiale gefördert werden. Bemerkenswert ist schließlich, daß der Bundesrat ausdrücklich eine Öffnung der Regionalpolitik in Richtung Raumordnungspolitik und damit auch eine organisatorische Abstimmung für die im Vorjahr in die Vernehmlassung geschickten «Grundzüge der Raumordnung Schweiz» sucht. Über die anvisierte Koordination zwischen Raumordnungs- und Sektoralpolitik orientiert Anhang 3.

\section{Ausblick}

Die Regionalpolitik des Bundes ist - wie manches in der Schweiz - sparsam instrumentiert und auf einen breiten Konsens ausgerichtet. Die in den letzten Jahren realisierten Akzentverschiebungen sind bemerkenswert und dürfen als zweckmäßig bezeichnet werden. Allerdings wird im Umfeld eines scharfen nationalen und internationalen Standortwettbewerbs - die tatsächliche Wirksamkeit der Regionalpolitik sorgfältig zu verfolgen sein. Wissenschaft und Politik werden hier noch enger zusammenarbeiten müssen, um die notwendigen Evaluationen sicherzustellen.

Verdrängt und teilweise unbeliebt sind nach wie vor die übergeordneten Fragen der Gebietsorganisation. Realistischerweise kann nicht angenommen werden, daß hier wesentliche Bewegungen entstehen. Es geht also darum, sowohl bezüglich der Technologie- und Industriepolitik ${ }^{8}$ als auch für die noch anstehenden Fragen der Stadtpolitik mit den bestehenden Trägern auszukommen. Das Beispiel der Hochschulpolitik (die nicht nur aus finanziellen Gründen im Umbruch begriffen ist) scheint zu zeigen, $\mathrm{da} ß$ in der interkantonalen Zusammenarbeit durchaus eine wichtige Erneuerungskraft gesehen werden kann. So oder so wird die Regionalpolitik des Bundes im Spannungsfeld vom Strukturwandel, Revitalisierung und Haushaltssanierung eine schwierige Kunst bleiben. 


\section{Anmerkungen}

1 Vgl. dazu S. BIERI, Schweizerische Regionalpolitik: Förderung, Umverteilung ... oder was?, DISP, Nr. 111, Zürich 1992, S. $25 \mathrm{ff}$., und die dort angegebene Literatur.

${ }^{2}$ Vgl. insbesondere R. L. FREY, Städtewachstum - Städtewandel, Basel und Frankfurt a. M. 1990.

${ }^{3} \mathrm{~A}$. ROSSI, Der wirtschaftliche Strukturwandel und die Regionen, Zürich 1995.

${ }^{4}$ Vgl. B. HOTZ-HART und C. KÜCHLER, Wissenschaft und Technologie im schweizerischen Innovationssystem, Mitteilungsblatt für Konjunkturfragen Nr. 2/95, Bern 1995, S. 14 ff.; $\mathrm{S}$. BIERI, Technologietransfer und Forschungszusammenarbeit: liberale Alternativen, in: B. B. GEMPER, Internationale Koordination und Kooperation, Hamburg 1990, S. 65 ff.; H. ERNSTE and V. MEIER (ed.), Regional Development and Contemporary Industrial Response, London and New York 1992.

${ }^{5}$ ROREP, Im Hinblick auf Europa 1993: Vorschläge für eine strategische schweizerische Regionalpolitik, Bern 1993. Eine sorgfältige Analyse traditioneller und "neuer" Wirtschaftsförderung bieten S. FORSTER et C. JEANRENAUD, Bilan 93: La promotion économique neuchâteloise, Neuchâtel 1994.

${ }^{6}$ Eidgenössisches Finanzdepartement, Neuer Finanzausgleich, Vernehmlassungsunterlage, Bern 1996.

${ }^{7}$ Botschaft Nr. 96.021.

${ }^{8}$ Bezüglich der Zusammenhänge zwischen räumlichen und industriellen Systemen: H. KILPER, Konzepte und Strategien regionaler Entwicklungen, Gelsenkirchen 1991; N. HANSEN, Do Producer Services Induce Regional Economic Development?, Journal of Regional Science, Vol. 30, Philadelphia 1990, S. 465 ff.

\section{Anhang 1}

Bundesgesetz über Investitionshilfe für Berggebiete (28.6.1974)

Art der Hilfe

Darlehen, Bürgschaften, Zinszuschüsse zur Restfinanzierung von Infrastrukturprojekten sowie von Land für Industrieund Gewerbezwecke

\section{Empfänger}

Gemeinden, öffentlich-rechtliche Körperschaften, Private (z. T.)

Voraussetzungen

- Gleichwertige Leistung des Kantons

- Genehmigtes regionales Entwicklungskonzept

- Projekt im Entwicklungskonzept begründet

- Finanzierungslücke

\section{Finanzierung}

Fonds de roulement

500 Mio. +300 Mio. Franken

+800 Mio. (bis 2000)
Anhang 2

Bundesbeschluß über Finanzierungsbeihilfen zugunsten wirtschaftlich bedrohter Regionen (17.6.1994)

Art der Hilfe

- Bürgschaften, Zinskostenbeiträge, Steuererleichterungen für Innovations- und Diversifikationsvorhaben (Investitionen)

- Beiträge an Informationsstellen für Innovationsvorhaben

Empfänger

- Industrielle oder gewerbliche Unternehmungen

Voraussetzungen

- Arbeitsplätze schaffen oder erhalten

- Angemessene Beteiligung der Unternehmung mit Eigenmitteln

- Kantonsbeteiligung

- Bankenbeteiligung

Finanzierung

- Rahmenkredit von insgesamt 20 Mio. Franken für Zinskostenbeiträge

- Bürgschaftsverpflichtung des Bundes insgesamt 300 Mio. Franken

Anhang 3

Koordination zwischen Raumordnungs- und Sektoralpolitik (Vorschlag des Bundesrates)

\begin{tabular}{|c|c|}
\hline Maßnahme & Bemerkungen \\
\hline $\begin{array}{l}\text { Zusammenlegung des } \\
\text { Ausschusses Investitions- } \\
\text { hilfe und der RPK und } \\
\text { Umbenennung in ROK. }\end{array}$ & $\begin{array}{l}\text { Faktisch vollzogen und vom } \\
\text { BR am 18. Dezember } 1995 \\
\text { sanktioniert. Auftrag an } \\
\text { Bundesämter, dem BR die } \\
\text { formell-rechtliche Abstüt- } \\
\text { zung noch vorzulegen. } \\
\text { Termin: bis Ende } 1996 . \\
\end{array}$ \\
\hline $\begin{array}{l}\text { Aufhebung der Koordina- } \\
\text { tionsweisungen und Rege- } \\
\text { lung der raumordnungs- } \\
\text { politischen Koordination in } \\
\text { einer Verordnung. } \\
\text { Gleichzeitig Ersatz der bis- } \\
\text { herigen Bundesratsaus- } \\
\text { schüsse "Raumordnung" } \\
\text { und "Regionalpolitik» durch } \\
\text { einen Ausschuß "Raum- } \\
\text { ordnungspolitik». }\end{array}$ & $\begin{array}{l}\text { Vom BR nach Vorliegen } \\
\text { der definitiven Grundzüge } \\
\text { der Raumordnung und } \\
\text { der parlamentarischen } \\
\text { Beschlüsse zur Neu- } \\
\text { orientierung der Regional- } \\
\text { politik zu beschließen. } \\
\text { Termin: voraussichtlich } \\
\text { Ende } 1996 .\end{array}$ \\
\hline $\begin{array}{l}\text { Überführung der Beraten- } \\
\text { den Kommission für regio- } \\
\text { nale Wirtschaftsförderung } \\
\text { und u. U. auch des For- } \\
\text { schungsstabes für Raum- } \\
\text { planung in einen Rat für } \\
\text { Raumordnung. }\end{array}$ & $\begin{array}{l}\text { Vom BR nach Antrag } \\
\text { des BRP und Biga } \\
\text { zu beschließen. } \\
\text { Termin: per 1.1.97. }\end{array}$ \\
\hline $\begin{array}{l}\text { Stärkung des interdeparte- } \\
\text { mentalen und amtsüber- } \\
\text { greifenden Kooperations- } \\
\text { und Koordinationsnetzes } \\
\text { (Modell der Verbindungs- } \\
\text { verantwortlichen). }\end{array}$ & Auf 1997 einzuführen. \\
\hline
\end{tabular}

